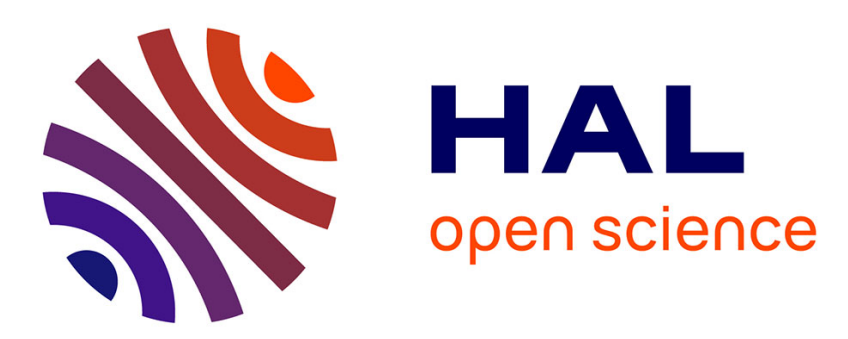

\title{
Association Optimization in Wi-Fi Networks based on the Channel Busy Time Estimation
}

\author{
Mohammed Amer, Anthony Busson, Isabelle Guérin-Lassous
}

\section{To cite this version:}

Mohammed Amer, Anthony Busson, Isabelle Guérin-Lassous. Association Optimization in Wi-Fi Networks based on the Channel Busy Time Estimation. IFIP Networking 2018, May 2018, Zürich, Switzerland. hal-01965313

\section{HAL Id: hal-01965313 https://hal.inria.fr/hal-01965313}

Submitted on 1 Apr 2020

HAL is a multi-disciplinary open access archive for the deposit and dissemination of scientific research documents, whether they are published or not. The documents may come from teaching and research institutions in France or abroad, or from public or private research centers.
L'archive ouverte pluridisciplinaire HAL, est destinée au dépôt et à la diffusion de documents scientifiques de niveau recherche, publiés ou non, émanant des établissements d'enseignement et de recherche français ou étrangers, des laboratoires publics ou privés. 


\title{
Association Optimization in Wi-Fi Networks based on the Channel Busy Time Estimation
}

\author{
Mohammed Amer, Anthony Busson, Isabelle Guérin Lassous
}

April 1, 2020

\begin{abstract}
With the centralized management paradigm offered by the recent IEEE 802.11 products, it is now easier and more efficient to optimize associations between access points (APs) and stations. Most of the optimization approaches consider a saturated network. Even if such traffic conditions are rare, the optimization of the association step under this assumption has the benefit to fairly share the bandwidth between stations. Nevertheless, traffic demands may be very different from one station to another and it may be more useful to optimize associations according to the stations' demands. In this paper, we propose an optimization of the association step based on the stations' throughputs and the channel busy time fraction (BTF). The latter is defined as the proportion of time the channel is sensed busy by an AP. Associations are optimized in order to minimize the greatest BTF in the network. This original approach allows the Wi-Fi manager/controller to unload the most congested AP, increase the throughput for most of the stations, and offer more bandwidth to stations that need it. We present a local search technique that finds local optima to this optimization problem. This heuristic relies on an analytical model that predicts BTF for any configuration. The model is based on a Markov network and a Wi-Fi conflict graph. NS-3 simulations including a large set of scenarios highlight the benefits of our approach and its ability to improve the performance in congested and non-congested Wi-Fi networks.
\end{abstract}

\section{Introduction}

Wireless LANs can offer the possibility to mobile devices to access the Internet. In particular, due to its efficiency and facility of deployment, IEEE 802.11 (referred as WiFi hereafter) has become a very popular wireless technology [1]. The density of access points (APs) allows the users to experience a high throughput and to be mobile without significant degradation of the link quality or connection interruption. Nevertheless, the limited number of non-overlapping channels makes difficult to ensure a good quality of service to users in dense WLANs without a dynamic and rational management. The management functions include channel assignment to access points, transmission power control, association between stations and APs, handovers, etc. In this work, we focus on the association. In Wi-Fi networks, association is the first step that allows a station to connect to the network. A station associates with an AP within its transmission range. If several APs are available in this area, the station will associate, generally, to the AP with the best RSSI (Radio Signal Strength Indicator). This metric, that measures the link quality between APs and stations, does not take into account the number of already associated stations neither the traffic load on the APs. It may lead to a heterogeneous distribution of stations among APs and consequently a bad distribution of the load in the network. Resources are therefore not optimally utilized, penalizing the overall performance of the network.

This problem and the need to facilitate AP administration have led to a centralization of the management in WiFi networks [2,3]. A Wi-Fi controller is in charge of all operations in the Wi-Fi network. It has a global vision of the network that enables a simple, flexible and efficient 
management of the resources. In particular, associations optimizing the resource usage can be computed in real time. The controller has then the ability to move stations from an AP to another using, for instance, the BSS transition management frames defined in IEEE 802.11v [4].

Most of the existing solutions to optimize associations propose solutions that aim to improve the overall network throughput and/or the fairness between stations in a saturated scenario [5-7]. The saturated scenario corresponds to a case where devices (stations and/or APs) have always a frame to send. This assumption is unrealistic but allows to express the minimum amount of throughput a device can obtain. Nevertheless, it does not take into account real traffic demands and the proposed association may be inaccurate. For example, if a part of the stations have very low traffic, the unused bandwidth can be reused by other stations with higher demands. A fair distribution of the resources may then be counter-productive: low traffic stations may be associated to the same AP which may be consequently idle whereas stations requiring high throughput are associated to overloaded APs.

In this paper, we propose an association optimization based on traffic demands. These traffic demands are defined as the downlink traffic from APs to stations and are measured in real time. The load of an AP is estimated through the busy time fraction (BTF). BTF corresponds to the fraction of time an AP senses the medium/channel busy due to its own transmissions or the ones from the other APs in its sensing range. This quantity is easily collected from the local Wi-Fi card statistics obtained on the current configuration. From a protocol point of view, it can be collected from the channel load request/report defined in IEEE $802.11 \mathrm{k}$ amendment [8]. In order to forecast its values for other configurations, we propose an analytical model that estimates BTF. It is based on a Markov network, a conflict graph and the current traffic demands. Our optimization problem consists in finding the association that minimizes the greatest BTF in the network. This original approach allows the Wi-Fi controller to unload the most congested AP and offer more bandwidth to stations that needs it. Our solution is evaluated with NS-3 simulations that cover a large set of scenarios (ISM and UNII bands, existing topologies of Wi-Fi networks and random ones, different number of stations and input rates, TCP and UDP flows, etc.). The obtained results highlight the benefit of our approach and its ability to improve per- formance in congested and non-congested networks.

The paper is organized as follows. In Section 2, we present the related work and our own contributions. The network model is described in Section 3.1. The BTF is defined in Section 3.2. The model estimating its value for any configuration is presented in the same section. Section 4 introduces the optimization problem and the heuristic used to propose approximate solutions. Numerical results are shown and discussed in Section 5. We conclude in Section 6.

\section{Related work}

The densification of Wi-Fi networks and the turn to its centralized management have motivated researches to optimize Wi-Fi configurations. Association between stations and APs is one of the key elements to improve the network performance. Below, we briefly summarize studies that address AP association. Contributions are classified as distributed, centralized and on-line.

Several approaches have proposed a distributed strategy. For instance in [9], the problem of AP association in WLAN is formulated through a mixed strategic game with a utility function that maximizes the throughput. They propose to users to move from their positions to improve their throughput. Distances traveled to a new AP are incorporated as a cost in the strategy game. The authors in [10] propose a solution for differentiated access service selection based on network applications, which are classified into four types according to their QoS requirements. Their approach can be used in a periodic or aperiodic strategy. In [11] a utility-based strategy is proposed to select the best AP according to the distance, data rate and delay. These three metrics are normalized between zero and one. Then, an equal weight is given to each metric within the utility function. The AP with the highest utility value is selected. Authors of [12] propose an algorithm that evaluates applications used by the stations, classified as data or voice, and changes the association accordingly. To achieve load balancing and good voice quality, the number of nodes connected to an AP and its RSSI are also considered in the association algorithm. However, evaluation is performed through a simulation of the model and not with a realistic network simulator. Moreover, one single data rate is considered for all transmis- 
sions between stations and APs. All these approaches assume only the saturated mode.

Centralized association has been proposed in order to achieve a global optimum. Wong et al. [13] propose a centralized max-min user throughput approach to optimize the AP re-association subject to a certain handover cost constraint. A multi-objective optimization function that maximizes the download user throughput and minimizes the number of handovers in saturated mode is also proposed in [14]. In [15], the authors formulate this problem as a non-cooperative game where each user tries to minimize its cost function, defined as the data transfer time. Their solution can be centralized or distributed. Authors of [16] propose a centralized approach to improve users' throughput in dense WLAN. They use signalinterference-noise-ratio (SINR) between APs and stations to control the association. In order to further coordinate interference and increase spatial reuse, an algorithm is proposed to adjust the clear channel assessment (CCA) threshold of the 802.11 MAC protocol. Taking into account the propagation environment, the authors of [17] investigate the impact of the AP deployments and station association in dense WLAN on the aggregate throughput.

On-line approaches have also been proposed in the literature. It consists in changing associations in real time, typically when an event occurs such as the arrival or departure of a station. In [18] the authors present a new AP selection metric. Their mechanism tries to maximize stations throughput as well as minimize its negative effect on high rate stations currently accommodated by the AP to which it wishes to associate. They propose two selection schemes based on this metric: a static one where stations only consider their association as well as a dynamic scheme where all associations are reevaluated from time to time. To improve the overall WLAN performance, Babul et al. propose in [19] an approach that considers simultaneously the channel assignment and the association control. However, validation is made through a simulation of the model and realistic Wi-Fi/network layers are not taken into account. Based on the Markov model to estimate the uplink and downlink throughput of clients, the authors of [20] propose an on-line AP association algorithm for 802.11n WLANs with heterogeneous clients. In this approach, authors seek to improve the overall network throughput.

All the cited approaches consider a saturated network except in [16] where the SINR is measured and used to determine data and error rates. Moreover, the traffic demand is not taken into account in the associations. The motivation of this paper is to design association algorithms able to adapt to traffic demands. It allows the controller to balance the load according to the real traffic, alleviate congested AP, and offer bandwidth to stations that need it. It is based on measurements available on most of the Wi-Fi products (e.g., busy time, data rates, error rates, etc.).

\section{System Model}

\subsection{Network Model}

We consider a general 802.11 WLAN consisting of a fixed number of APs. The set of APs is assumed to belong to the same extended service set (ESS) and is managed by a WLAN controller. We take into account only downlink traffic, from the APs to the stations, as downlink traffic is preponderant compared to uplink traffic [21]. The controller is in charge of determining the association. When a new station connects to the ESS, it first associates with the default AP which is, for most of the implementation, the one with the best RSSI. The controller can, according to our algorithm, change associations at regular intervals or when a particular event occurs (arrival/departure of stations for instance). We assume that the controller collects periodically the following measurements from APs:

- the current association,

- the busy time fraction for each AP,

- the conflicts between APs (the conflict graph is formally defined later in this paper),

- for each station:

- the data rates between APs and the station,

- the throughput and the average frame size received by the station from its AP,

- the error rate (or equivalently the probability of success) between the station and its AP.

It is worth noting that most of these measurements are already available on most of the AP products (e.g., Cisco Aironet Series APs). 
When the controller finds out a better association, it triggers the corresponding changes: through control frames, stations can be disassociated from the current AP and associated to the new one. The application of a new configuration induces a re-association cost. The condition for applying a new association may be function of the cost and gain of the new configuration.

The association, proposed in this work, is based on the estimation of BTF. The BTF of the current configuration is known, but it has to be predicted for the other configurations that can be considered for a new association. Our prediction model relies on the following assumptions:

- Data rate: APs are able to determine the best data rate for all the stations in its transmission range (associated or not).

- Throughput: we assume that a station, associated to a new AP, will request at least the same throughput as in the current configuration.

- Probability of success: the probability of success for each station (probability that a frame is correctly received) is measured between APs and their associated stations. Its prediction for another association is difficult. In our model, we assume that this probability remains the same if the station does not change its channel when it reassociates. In case of a channel change, the success probability is set to the smallest probability of success among the stations already associated with the new AP.

The objective function based on BTF and the heuristic used to minimize it are presented in Section 4. We introduce, in the next section, the analytical model used to estimate BTF for all APs.

\subsection{Busy Time Fraction estimation}

BTF for an AP is defined as the fraction of time the channel is sensed occupied. This measurement can be obtained from the measurement reports of IEEE $802.11 \mathrm{k}$ or directly from the physical registers of the interface that measures the busy time according to the CCA mechanism [1]. This quantity is generally available for the current association. But in the context of our optimization, it is necessary to estimate this fraction for any other configuration.
In our model, we define $b_{j}$ the busy time fraction for an AP $j$. This time is composed of two quantities: the local busy time fraction and the neighbor busy time fraction. The local busy time fraction, denoted $b_{j}^{L}$, corresponds to the time, per second, the channel is occupied by its own transmissions. This time takes into account the physical occupation of the channel and the access method times (back-off, DIFS, etc.). The neighbor busy time fraction, denoted $b_{j}^{N}$, is the proportion of time the channel is occupied by APs in its sensing range. It considers only the physical occupation of the channel corresponding to transmissions. We get,

$$
b_{j}=b_{j}^{L}+b_{j}^{N}
$$

\subsubsection{Local Busy Time Fraction}

This time includes the time to transmit data on the physical channel $\left(T_{P H Y}\right)$ to one of its stations and the time of the access method $\left(T_{M A C}\right)$. Thus, the local BTF of an AP $j$ is the sum of the busy time fractions due to transmissions to all of its stations.

$$
b_{j}^{L}=\sum_{i \in S_{j}} b_{i j}^{L}
$$

where $S_{j}$ is the set of stations associated with $\mathrm{AP} j$, and $b_{i j}^{L}$ the BTF corresponding to the transmissions from AP $j$ to station $i$. $b_{i j}^{L}$ can be computed as follows:

$$
b_{i j}^{L}=\overline{T\left(R_{i j}, L\right)} \times D_{i}
$$

where $\overline{T\left(R_{i j}, L\right)}$ is the average time required for $\mathrm{AP} j$ to successfully transmit a frame of size $L$ to station $i$ with data rate $R_{i j} . D_{i}$ is the average number of datagrams transmitted to station $i$ in one second. It does not take into account retransmissions.

Nevertheless, a datagram is subject to transmission errors and may require one or more retransmissions. According to the IEEE 802.11 standard, the time required for AP $j$ to successfully transmit a frame of size $L$ to station $i$ at data rate $R_{i j}$ after $k$ attempts is given by:

$$
\begin{aligned}
& T\left(k, R_{i j}, L\right)=T_{P H Y}+T_{M A C} \\
& T_{P H Y}=T_{P}+T_{H}+L / R_{i j}+T_{A c k} \\
& T_{M A C}=T_{D I F S}+T_{S I F S}+T_{\text {backoff }}(k)
\end{aligned}
$$


$T_{P}$ and $T_{H}$ represent the duration of the preamble and the header of the physical layer. $T_{D I F S}$ is the DCF Inter Frame Space and $T_{S I F S}$ is the Short Inter Frame Space. $T_{A C K}$ is the duration of the ACK frame. $T_{\text {backoff }}(k)$ is the average back-off after $k$ unsuccessful successive transmission attempts and is given by:

$$
T_{\text {backoff }}(k)=\min \left(\frac{2^{k}\left(C W_{\min }+1\right)-1}{2}, C W_{\max }\right) \times T_{\text {slot }}
$$

where $T_{\text {slot }}$ is the duration of a slot. $C W_{\min }$ and $C W_{\max }$ are respectively the minimum and maximum sizes of the contention window.

The average time that $\mathrm{AP} j$ requires to correctly transmit a single datagram to station $i$ is [22]:

$$
\begin{gathered}
\overline{T\left(R_{i j}, L\right)}=p_{i j} T\left(0, R_{i j}, L\right)+ \\
\sum_{k=1}^{m}\left(p_{i j}\left(1-p_{i j}\right)^{k}\left(\sum_{l=0}^{k-1} T_{c}\left(l, R_{i j}, L\right)+T\left(k, R_{i j}, L\right)\right)\right)
\end{gathered}
$$

where $m$ is the maximum number of retransmissions, $p_{i j}$ is the probability of success to transmit from $\mathrm{AP} j$ to station $i$ and $T_{c}\left(l, R_{i j}, L\right)=T_{\text {backoff }}(l)+T_{D I F S}+T_{P}+T_{H}+$ $L / R_{i j}+T_{A T O}$ is the time between two consecutive transmissions if the frame transmission fails $\left(T_{A T O}\right.$ is the acknowledgment timeout).

Note that, in the previous formula, the value of the parameter $R_{i j}$ may be different at each retransmission, but is constant during a retransmission. It is consistent with current implementations like Minstrel.

\subsubsection{Neighbor Busy Time Fraction}

In this section, we present the model to estimate the fraction of time the channel is sensed busy by an AP due to the activity of the other APs. Formally, in Wi-Fi networks, channel sensing is performed by the CCA (Clear Channel Assessment) mechanism [1]. We consider a conflict graph [23] composed of vertices that represent APs and where an edge $(j, k)$ exists if APs $j$ and $k$ detect their mutual transmissions (according to the CCA mechanism).

To compute the Neighbor BTF $b_{j}^{N}$, we introduce a set of notations. We define the event $A_{i}$ as follows:

$$
A_{i}=\{\mathrm{AP} i \text { is transmitting }\}
$$

So, the fraction of time that the channel is sensed busy by AP $j$ due to transmissions from its neighbors is given by:

$$
b_{j}^{N}=\operatorname{Pr}\left(\bigcup_{i \in N_{j}} A_{i}\right)
$$

where $N_{j}$ is the set of neighbors of vertex $j$ in the conflict graph. The events $A_{i}$ are not disjoint and the computation of the union is consequently not trivial. To compute this probability, we propose to use the Inclusion-Exclusion Principle [24], which is defined as:

$$
\operatorname{Pr}\left(\bigcup_{i \in N_{j}} A_{i}\right)=\sum_{k=1}^{\left|N_{j}\right|}\left((-1)^{k-1} \sum_{\substack{I \subset N_{j} \\|I|=k}} \operatorname{Pr}\left(\bigcap_{l \in I} A_{l}\right)\right)
$$

where $I \subset N_{j}$ with $|I|=k$ describes all the subsets of $N_{j}$ with cardinal $k$.

If $|I|=1$, the computation $\operatorname{Pr}\left(\bigcap_{l \in I} A_{l}\right)$ is trivial. When $|I|>1$, we have to take into account the conflict graph. Indeed, there are two possible cases that we illustrate through the example given in Figure 1. We consider BTF of AP 1. It has three neighboring APs. As there is a link between AP 2 and AP 3, they cannot transmit at the same time and $\operatorname{Pr}\left(A_{2} \cap A_{3}\right)=0$. As there is no conflict between AP 3 and AP 4, transmissions from these APs can overlap and $\operatorname{Pr}\left(A_{3} \cap A_{4}\right) \neq 0$.

Consequently if two APs in $I$ are neighbors, then their transmissions are exclusive and the intersection is zero:

$$
\operatorname{Pr}\left(\bigcap_{l \in I} A_{l}\right)_{\substack{I \subset N_{j} \\|I|=k}}=0 \text {, if } \exists(p, q) \in I^{2} \text { s.t. } p \in N_{q}\left(q \in N_{p}\right)
$$

Otherwise, transmissions are not exclusive and this probability may be $>0$.

But, the events $\left(A_{l}\right)_{l \in I}$ are not independent and the probability of their intersection (overlap) cannot be computed as their product. In order to compute this probability, we consider this problem as an Undirected Graphical Model or Markov Network. It is based on a graph where the vertices correspond to the events $A_{i}$ and the edges represent the dependencies between them. The considered graph is then the same as the APs conflict graph. 


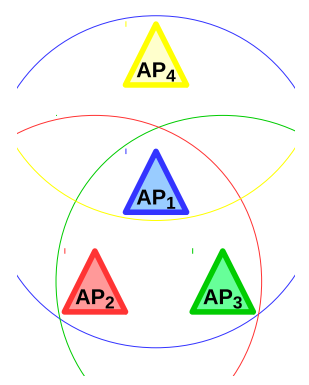

(a) APs Topology

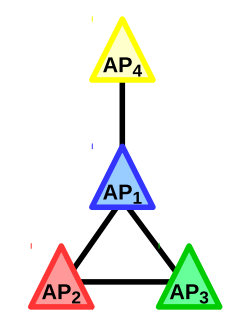

(b) APs conflict graph

Figure 1: Topology with 4 APs and its conflict graph

In Figure 2 we show the previous example with a topology with 4 APs conflict graph and the corresponding Markov Network.

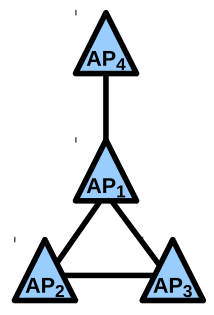

(a) APs conflict graph

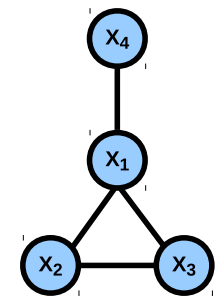

Figure 2: 4 APs conflict graph and its Markov Network. Formally, the Markov Network is defined as function of the correlations between random variables. So, we introduce the random variables $\left(X_{j}\right)_{j}$ which indicates if $\mathrm{AP} j$ is transmitting $\left(A_{j}=\left\{X_{j}=1\right\}\right)$.

Markov network relies on the Global Markov Property [25], which is defined as follows:

Definition: For any disjoint subsets of the vertices $A$, $B$, and $C$ in the graph $G$ such that $C$ separates $A$ and $B$ (i.e. every path between a node in $A$ and a node in $B$ passes through a node in $C$ ), the random variables
$X_{A}$ are conditionally independent of $X_{B}$ given $X_{C}$, i.e. $X_{A} \perp X_{B} / X_{C}$, where $X_{A}=\left\{X_{v}\right\}_{v \in A}$.

In our context, we assume that the transmissions of non-neighboring APs are independent if the set of all their neighbors (union of neighbors) does not transmit:

$$
\begin{gathered}
\operatorname{Pr}\left(\bigcap_{l \in I} A_{l}\right)=\operatorname{Pr}\left(\bigcap_{l \in I} A_{l} \mid \bigcap_{l^{\prime} \in I^{\prime}} \overline{A_{l^{\prime}}}\right) \operatorname{Pr}\left(\bigcap_{l^{\prime} \in I^{\prime}} \overline{A_{l^{\prime}}}\right), \\
I \subset N_{j},|I|=k, I^{\prime}=\bigcup_{i \in I} N_{i}
\end{gathered}
$$

Applying this property, we obtain:

$$
\begin{aligned}
\operatorname{Pr}\left(\bigcap_{l \in I} A_{l}\right)= & \prod_{l \in I}\left(\operatorname{Pr}\left(A_{l} \mid \bigcap_{l^{\prime} \in I^{\prime}} \overline{A_{l^{\prime}}}\right)\right) \operatorname{Pr}\left(\bigcap_{l^{\prime} \in I^{\prime}} \overline{\overline{A_{l^{\prime}}}}\right) \\
= & {\left[\prod_{l \in I} \frac{\operatorname{Pr}\left(A_{l} \cap\left(\bigcap_{l^{\prime} \in I^{\prime}} \overline{A_{l^{\prime}}}\right)\right)}{\operatorname{Pr}\left(\bigcap_{l^{\prime} \in I^{\prime}} \overline{A_{l^{\prime}}}\right)}\right] \operatorname{Pr}\left(\bigcap_{l^{\prime} \in I^{\prime}} \overline{A_{l^{\prime}}}\right) } \\
= & \frac{\prod_{l \in I} \operatorname{Pr}\left(A_{l} \cap\left(\bigcap_{l^{\prime} \in I^{\prime}} \overline{A_{l^{\prime}}}\right)\right)}{\left(\operatorname{Pr}\left(\bigcap_{l^{\prime} \in I^{\prime}} \overline{A_{l^{\prime}}}\right)\right)^{|I|-1}}
\end{aligned}
$$

Moreover, we have,

$$
\begin{aligned}
\operatorname{rClPr}\left(A_{l} \cap\left(\bigcap_{l^{\prime} \in I^{\prime}} \overline{A_{l^{\prime}}}\right)\right)= & \operatorname{Pr}\left(A_{l} \cup\left(\bigcup_{l^{\prime} \in I^{\prime}} A_{l^{\prime}}\right)\right) \\
& -\operatorname{Pr}\left(\bigcup_{l^{\prime} \in I^{\prime}} A_{l^{\prime}}\right)
\end{aligned}
$$

and,

$$
\operatorname{Pr}\left(\bigcap_{l^{\prime} \in I^{\prime}} \overline{A_{l^{\prime}}}\right)=1-\operatorname{Pr}\left(\bigcup_{l^{\prime} \in I^{\prime}} A_{l^{\prime}}\right)
$$


By substituting (6) and (7) in Equation (5), we obtain:

$$
\operatorname{Pr}\left(\bigcap_{l \in I} A_{l}\right)=\frac{\prod_{l \in I}\left(\operatorname{Pr}\left(\bigcup_{l^{\prime} \in I^{\prime} \cup\{l\}} A_{l^{\prime}}\right)-\operatorname{Pr}\left(\bigcup_{l^{\prime} \in I^{\prime}} A_{l^{\prime}}\right)\right)}{\left(1-\operatorname{Pr}\left(\bigcup_{l^{\prime} \in I^{\prime}} A_{l^{\prime}}\right)\right)^{|I|-1}}
$$

We obtain a system of nonlinear equations where each term (variable) is the probability of union of the events $\left\{A_{i}\right\}$. As the number of possible combinations between all events is finite then the system contains a finite number of equations. This system can be solved by any numerical method.

To sum up, $b_{j}^{N}$, given by Equation (2), is obtained from the union of the events $A_{i}$ (Equation (3)), itself obtained from Equation (8).

\section{Association optimization}

Our association scheme is based on BTF. This quantity describes the saturation level of an AP. If an AP is saturated, its BTF is close to 1 , and the associated stations are likely restrained in terms of throughput and thus unsatisfied. On the other hand, if BTF is lower, stations necessarily obtain the required throughput since a part of the bandwidth is available and not used. Stations are then assumed satisfied in terms of their throughput demand. The optimization problem aims to minimize the maximum BTF in the network. Formally, it is given by Equation (9).

$$
\text { minimize } \max _{j \in A}\left[b_{j}\right]
$$

where $A$ is the set of APs and $b_{j}$ the BTF of AP $j$. This objective function has been designed to:

- share the load among APs as it will try (if such solutions exist) to unload the most loaded APs,

- satisfy a maximum of stations in terms of throughput, as it will try to decrease BTF of saturated APs,

- increase the station throughputs, as unsatisfied stations will be moved to unsaturated APs.

The evaluation of BTF of each AP relies on the model proposed in Section 3.2 which predicts $b_{j}$ (i.e. BTF of AP j) for any association. We propose an iterative heuristic based on the principle of local search to solve our optimization problem. Local search is an important class of heuristics used to solve combinatorial optimization problems. The key idea of a local search algorithm is to start from an initial feasible solution (association) and iteratively find, at each iteration, a solution called a best neighbor that improves the objective function [26]. The main benefits of local search lie in its simplicity and its iterative process which can stop the optimization process at any time to comply with a constraint like the computation time for instance. This is supported by the fact that the local search algorithms consider only complete feasible solutions during the search. The proposed algorithm has then the advantage to improve Wi-Fi associations at each iteration, and can be stopped at any time with a feasible solution. The time that the system spends in computing a solution can thus be bounded and tuned.

The controller runs the iterative local search Algorithm 1, which consists in:

1. starting with an initial configuration (RSSI or any current association),

2. then at each iteration, it chooses, among all possible association changes, the one that minimizes the objective function. This configuration becomes the current solution on which to apply the next iteration.

\section{Evaluation}

In this section, we present the simulation environment, the performance metrics, and the different simulation scenarios. We then discuss the simulation results.

\subsection{Simulation configuration}

We used a fixed point method [27] to solve the system of nonlinear equations. The optimization heuristic is implemented using $\mathrm{C}++$ programming language. Simulations have been performed with the network simulator 3 (ns-3). We use the log-distance path loss model. The transmission power is set to $16 \mathrm{dbm}$. The number of APs and stations is fixed for each topology. Stations are associated according to the RSSI value in the initial configuration. The ideal Wi-Fi manager of ns-3 is used to determine the 
data rates between APs and stations. In the figures, we increase the input rates (mean of the flow rates between APs and stations). For each input rate, simulations are repeated 30 times with different stations position. Flow rates are constant during a simulation but set randomly with a given average. Therefore, physical transmission rates and flows are different from a station to another for each simulation. A $95 \%$ confidence interval is computed over these 30 samples. The duration of each simulation is 60 seconds.

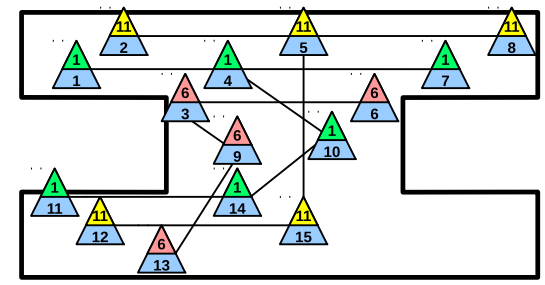

Figure 3: WLAN topology at one floor in our university. The upper number corresponds to the used channel and the lower number corresponds to the AP number.

\subsection{BTF Estimation}

In order to estimate the accuracy of the BTF model proposed in Section 3.2 we compare its values obtained by simulation and from the model. The considered topology is the WLAN of our university at a given floor of the building. This network is composed of 15 APs as shown in Figure 3. APs use the ISM frequency band (2.4 Ghz). In this band the number of non-overlapping channels is limited (three orthogonal channels: 1, 6 and 11). This Figure shows also the three conflict graphs (one for each orthogonal channel).

The simulated scenario consists of 60 stations randomly distributed in the coverage area of the 15 APs. We plot in Figure 4 the BTF values according to the average input rate (mean of the flow rates). To evaluate the effectiveness of the approach in dense environment, we consider BTF of APs 4 and 5 of our topology (each one is in conflict with 3 other APs).

For AP 4, the difference between the measured BTF (simulation) and model is $7 \%$ when the load is low $(B T F<0.2)$. This difference decreases to $2 \%$ when

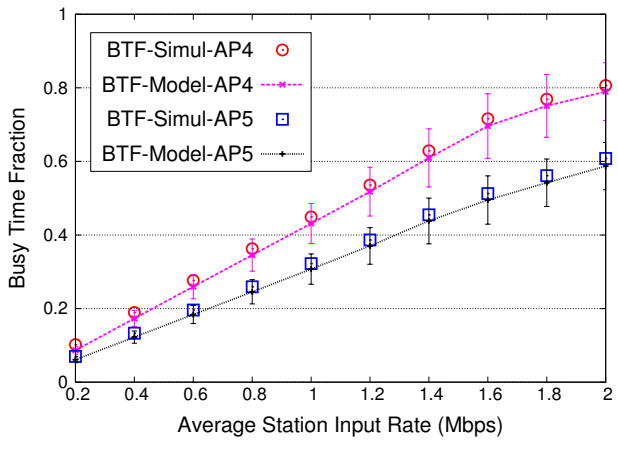

Figure 4: Simulation vs Model BTF values for APs 4 and 5 in the university topology

the load increases $(B T F>0.7)$. For AP 5 the difference varies in average between $3 \%$ and $6 \%$. According to these results, it appears that the model provides a very good estimation of BTF. The model slightly underestimates the BTF as we do not take into account acknowledgments transmitted by the stations. It is neglected because to include them in the BTF computation a full knowledge of the conflict graph is required (in particular conflicts between stations) whereas in our model only conflicts between APs are considered. It is more realistic from an implementation point of view, but these conflicts/acknowledgments can be easily integrated in the model if the controller is able to infer them.

\subsection{Association Optimization}

In order to evaluate the improvement brought by our approach, we have considered two different topologies. From the simulation results, we compute the following performance parameters:

- Busy Time Fraction: for each simulation we consider the greatest BTF in the network.

- Number of unsatisfied stations: it represents the proportion of stations that are not satisfied in terms of throughput (i.e. when the ratio between the obtained throughput and the demand is less than 98\%).

- Throughput Satisfaction Ratio: it is the ratio between the throughput obtained and the throughput requested for each station. 


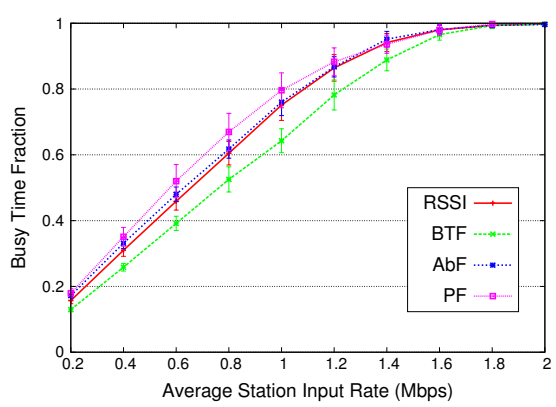

(a) BTF of the most loaded AP

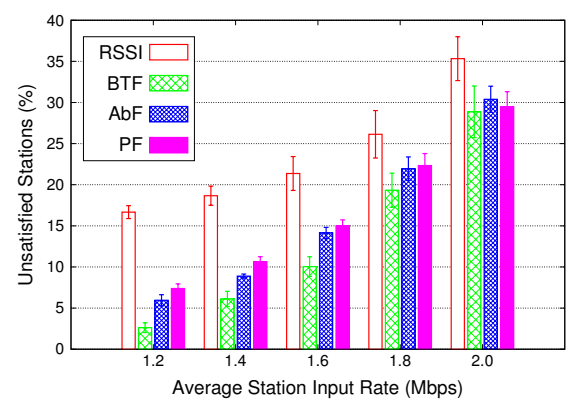

(b) Unsatisfied stations

Figure 5: BTF of the most loaded AP and proportion of unsatisfied stations according to the WLAN load.

Our solution is compared to three existing approaches:

- Initial configuration: stations associate to APs according to the value of the RSSI. It is denoted as RSSI in the figures.

- Access based Fairness [5]: stations associated to the same AP have the same opportunity of service in saturated mode. It is denoted AbF in the figures.

- Proportional Fairness [7]: the saturated mode is also considered with an access opportunity to the medium which is proportional to the data rate of each station. It is denoted PF in the figures.

In the performance evaluation we consider different flow types, as follows:

- UDP: all the packets have the same size (1500 bytes) and the inter-packet time is constant for each station.

- Real trace: packet sizes vary according to a distribution obtained from a real trace [28] (Average Packet Size $=755.572$ bytes and Standard Deviation $=674.05$ ).

- TCP: constant bit rate flows are installed over TCP connections.

ENS topology. The first scenario considers the topology of our university (ENS) used in the previous section and UDP flows. Figure 5a illustrates the BTF of the most loaded AP as function of the WLAN load. For AbF, the busy time fraction is approximately the same as the one observed for the RSSI association. For PF association, the busy time fraction is even increased of $11 \%$ in average. With BTF optimization, the busy time fraction is decreased by approximately $15 \%$ when the network is not heavily loaded. This will allow stations to request more traffic without saturating APs. It appears clearly that AbF and PF approaches are unable to decrease BTF in unsaturated WLAN.

Nevertheless, when the WLAN reaches saturation, the three approaches provide similar results in terms of busy time fraction. To show the benefit of our approach when the WLAN becomes loaded (more than 1 Mbps per station in the figure), we measure and compare the number of stations not satisfied before and after the optimization for the three approaches. Results are shown in Figure 5b. Our solution reduces the number of unsatisfied stations by more than $84 \%$ for an average load of $1.2 \mathrm{Mbps}$ per station, and $18 \%$ for an average load of $2 \mathrm{Mbps}$ per station. For $\mathrm{AbF}$, the gain varies between $64 \%$ and $14 \%$, and for PF it varies between 55\% and 16\%. Even in saturated conditions, our solution still presents a lower number of unsatisfied stations.

Random topologies. To evaluate our approach with denser topology and more complex conflict graphs between APs, we performed simulations on random topologies. Each topology is composed of 25 APs uniformly deployed in a square of size $500 \mathrm{~m} \times 500 \mathrm{~m}$. 100 stations are distributed in the coverage area of these APs. APs 


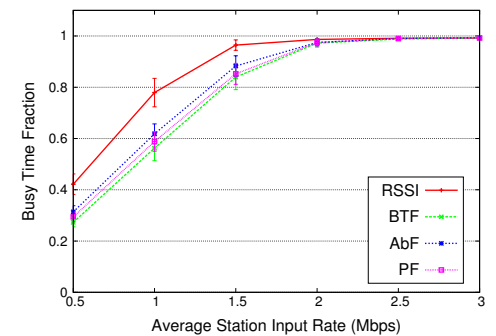

(a) BTF of the most loaded AP

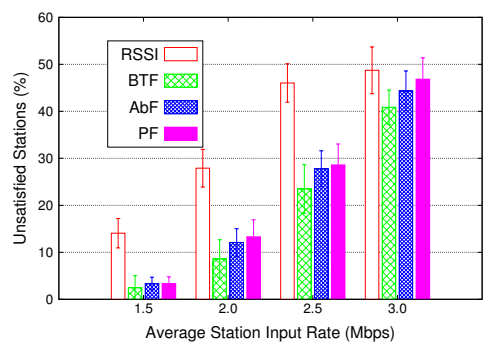

(b) Unsatisfied stations

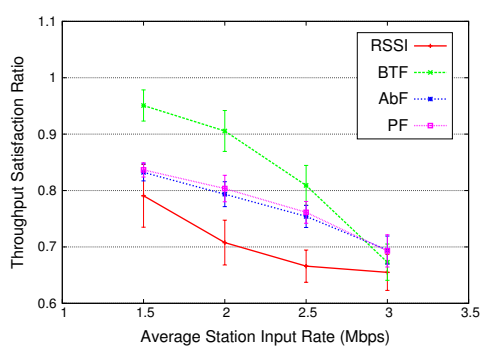

(c) Throughput Satisfaction Ratio

Figure 6: BTF association optimization using random topology with the Real Trace flows

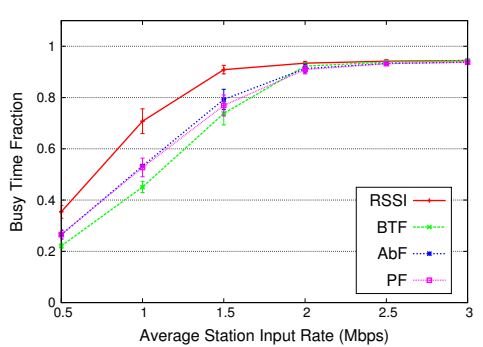

(a) BTF of the most loaded AP

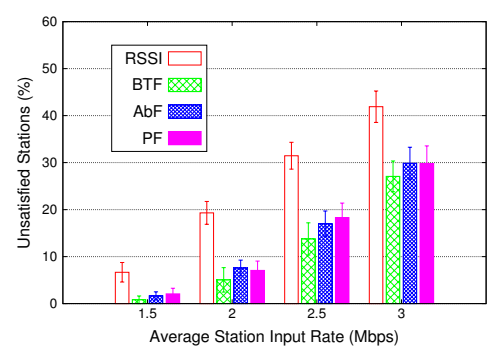

(b) Unsatisfied stations

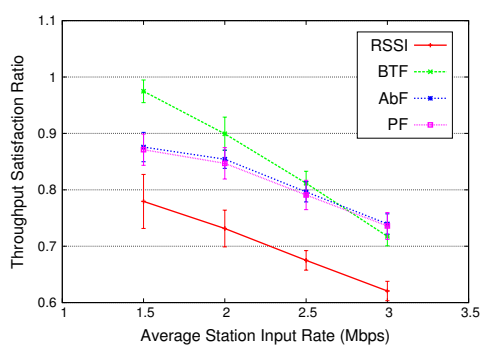

(c) Throughput Satisfaction Ratio

Figure 7: BTF association optimization using random topology with TCP flows

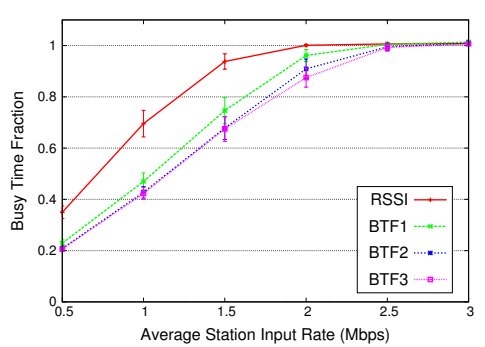

(a) BTF of the most loaded AP

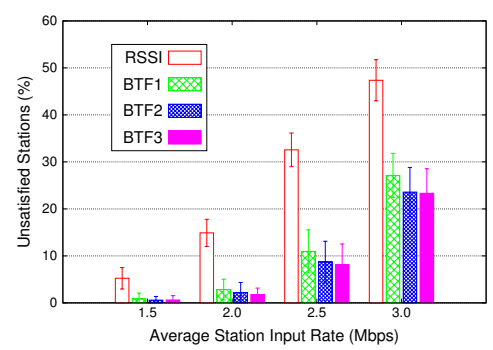

(b) Unsatisfied stations

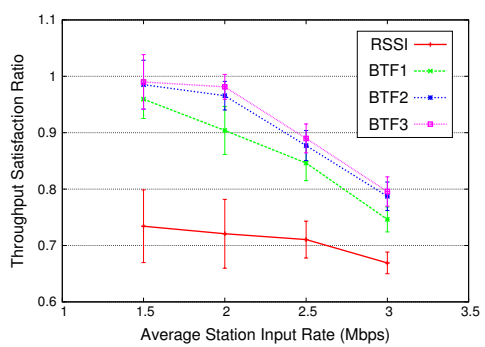

(c) Throughput Satisfaction Ratio

Figure 8: BTF association optimization using random topology with UDP flows and applied 3 times

are configured with 8 orthogonal channels. In this scenario, APs location is changed at each simulation. This randomness allows us to consider an important number of different topologies (30 for each set of parameters).

Figure 6 illustrates the results of the simulations with the real trace. It allows us to evaluate performances for different packet sizes. Figure 6a shows that the BTF approach can offload $35 \%$ of the most charged AP. AbF and $\mathrm{PF}$ approaches allow a load reduction of about $25 \%$ and $30 \%$ respectively. In Figure 6b, the number of unsatisfied stations is decreased of 54\% with BTF optimization with regard to to the RSSI association. For AbF and PF, 
improvements are $45 \%$ and $42 \%$ in average. The satisfaction ratio is shown in Figure 6c. For the BTF approach, it is increased in average between $3 \%$ and $27 \%$. On the other hand, AbF approach allows a gain between $6 \%$ and $13 \%$, and between $5 \%$ and $14 \%$ for PF.

Figure 7 shows results with TCP flows. In Figure 7a our approach decreases the load of the most loaded AP up to $37 \%$. For the $\mathrm{AbF}$ and $\mathrm{PF}$ approaches the decrease is almost the same and does not exceed $25 \%$. Regarding the number of unsatisfied stations shown in Figure $7 \mathrm{~b}$, BTF allows a gain between $87 \%$ and $35 \%$. For AbF, the decrease of the number of unsatisfied stations varies between $79 \%$ and $28 \%$. For PF, the decrease is between $69 \%$ and $29 \%$. Figure $7 \mathrm{c}$ plots the throughput satisfaction ratio. With BTF the stations gain in throughput on average between $25 \%$ and $15 \%$. The $\mathrm{AbF}$ and $\mathrm{PF}$ approaches allow an average gain of $17 \%$ and $16 \%$ respectively.

In order to illustrate the impact of the BTF approach in a more realistic implementation context where the optimization process is executed whenever needed (at regular interval for instance), we have simulated the same scenario with UDP flows in which our optimization method is applied 3 times.After each optimization we evaluate the performance and then collect the necessary measures for the next optimization. Results for this scenario are shown in Figure 8.

Even if the first optimization allows significant improvements for all performance parameters, the second and third optimizations can further improve these performances. For the greatest BTF in the network, the improvement for the first, second and third optimizations is in average $30 \%, 35 \%$ and $36 \%$ respectively. For the unsatisfied station number, the improvement is $68 \%, 74 \%$ and $75 \%$ respectively. For the throughput satisfaction ratio, the improvement is $22 \%, 27 \%$ and $29 \%$ respectively.

All these results tend to show that our solution generally offers better performance whatever the load of the network. Nevertheless, when the network is very loaded (average station input rate $>2.5 \mathrm{Mbps}$ ) the $\mathrm{AbF}$ and $\mathrm{PF}$ approaches allow to have results close to BTF.

\section{Conclusion}

In this paper, we propose an original approach for the association optimization in Wi-Fi networks. Our solution is based on a model predicting BTF at each AP and aims to associate stations in order to minimize the most loaded AP. We have shown through simulations that the model allows an accurate estimation of BTF in the considered configurations. Moreover, performance evaluation has shown that such an approach reduces the congestion in the network as it decreases the most loaded AP. This improvement can reach $18 \%$ in average when the network is not heavily loaded. Also, our solution decreases the number of unsatisfied stations, up to $80 \%$ when the network becomes saturated and improves throughput of the unsatisfied stations. When the network is unsaturated, which corresponds to the normal conditions of a Wi-Fi network, approaches based on models that rely on saturated conditions are significantly less efficient that our proposition.

\section{Acknowledgment}

The authors wish to thank Isabel Martin Faus for her thorough and constructive comments of an earlier version of this paper.

\section{References}

[1] "IEEE standard for information technologytelecommunications and information exchange between systems local and metropolitan area networks-specific requirements part 11: Wireless LAN medium access control (MAC) and physical layer (PHY) specifications," IEEE Std 802.11-2012, pp. 1-2793, March 2012.

[2] P. Calhoun, M. Montemurro, and D. Stanley, "Control and provisioning of wireless access points (CAPWAP) protocol specification," Internet Requests for Comments, RFC Editor, RFC 5415, March 2009.

[3] K. Sood, S. Liu, S. Yu, and Y. Xiang, "Dynamic access point association using software defined networking," in 2015 International Telecommunication Networks and Applications Conference (ITNAC), Nov 2015, pp. 226-231. 
[4] IEEE Std 802.11v-2011 - Amendment 8: IEEE 802.11 Wireless Network Management, pp. 1-433, Feb 2011.

[5] M. Amer, A. Busson, and I. Guérin Lassous, "Association optimization in wi-fi networks: Use of an access-based fairness," in Proceedings of the 19th ACM International Conference on Modeling, Analysis and Simulation of Wireless and Mobile Systems, ser. MSWiM '16. ACM, 2016, pp. 119126.

[6] H. Tang, L. Yang, J. Dong, Z. Ou, Y. Cui, and J. Wu, "Throughput optimization via association control in wireless LANs," Mobile Networks and Applications, vol. 21, no. 3, pp. 453-466, 2016.

[7] O. B. Karimi, J. Liu, and J. Rexford, "Optimal collaborative access point association in wireless networks," in IEEE INFOCOM 2014. Conference on Computer Communications, April 2014, pp. 11411149.

[8] IEEE 802.11k-2008 - Amendment 1: IEEE 802.11 Radio Resource Measurement, 2008.

[9] I. Sohn, "Access point selection game with mobile users using correlated equilibrium," PLOS ONE, vol. 10, no. 3, pp. 1-13, 032015.

[10] Z. Chen, Q. Xiong, Y. Liu, and C. Huang, "A strategy for differentiated access service selection based on application in wlans," in 2014 IEEE Conference on Computer Communications Workshops (INFOCOM WKSHPS), April 2014, pp. 317-322.

[11] J. B. Ernst, S. Kremer, and J. J. P. C. Rodrigues, “A utility based access point selection method for ieee 802.11 wireless networks with enhanced quality of experience," in 2014 IEEE International Conference on Communications (ICC), June 2014, pp. 23632368.

[12] M. V. Ramesh and M. S. Nisha, "Design of optimization algorithm for wlan ap selection during emergency situations," in 2011 IEEE 3rd International Conference on Communication Software and Networks, May 2011, pp. 340-344.
[13] W. Wong, A. Thakur, and S. H. G. Chan, "An approximation algorithm for ap association under user migration cost constraint," in IEEE INFOCOM 2016 - The 35th Annual IEEE International Conference on Computer Communications, April 2016, pp. 1-9.

[14] E. Zola, F. Barcelo-Arroyo, and A. Kassler, "Multiobjective optimization of wlan associations with improved handover costs," IEEE Communications Letters, vol. 18, no. 11, pp. 2007-2010, Nov 2014.

[15] K. Khawam, J. Cohen, P. Muhlethaler, S. Lahoud, and S. Tohme, "Ap association in a ieee 802.11 wlan," in 2013 IEEE 24th Annual International Symposium on Personal, Indoor, and Mobile Radio Communications (PIMRC), Sept 2013, pp. 21422147.

[16] P. B. Oni and S. D. Blostein, "Ap association optimization and cca threshold adjustment in dense wlans," in 2015 IEEE Globecom Workshops (GC Wkshps), Dec 2015, pp. 1-6.

[17] A. Ozyagci, K. W. Sung, and J. Zander, "Association and deployment considerations in dense wireless lans," in 2014 IEEE 79th Vehicular Technology Conference (VTC Spring), May 2014, pp. 1-5.

[18] M. Abusubaih, S. Wiethoelter, J. Gross, and A. Wolisz, "A new access point selection policy for multi-rate ieee 802.11 wlans," Int. J. Parallel Emerg. Distrib. Syst., vol. 23, no. 4, pp. 291-307, Aug 2008.

[19] B. P. Tewari and S. C. Ghosh, "Interference avoidance through frequency assignment and association control in ieee 802.11 wlan," in 2014 IEEE 13th International Symposium on Network Computing and Applications, Aug 2014, pp. 91-95.

[20] D. Gong and Y. Yang, "On-line ap association algorithms for 802.11n wlans with heterogeneous clients," IEEE Transactions on Computers, vol. 63, no. 11, pp. 2772-2786, Nov 2014.

[21] F. Malandrino, C.-F. Chiasserini, and S. Kirkpatrick, "Cellular Network Traces Towards 5G: Usage, Analysis and Generation," IEEE Transactions on Mobile Computing, vol. PP, no. 99, p. 1, August 2017. 
[22] V. Atanasovski and L. Gabrilovska, "Influence of header compression on link layer adaptation in ieee 802.11b," in 2005 International Conference on Wireless Networks, Communications and Mobile Computing, vol. 2, June 2005, pp. 1551-1556 vol.2.

[23] A. Busson, E. Fleury, and N. M. Phung, "A simple method to infer Wi-Fi conflict graph," in CORES2017, Quiberon, France, May 2017.

[24] T. Andreescu and Z. Feng, Inclusion-Exclusion Principle. Boston, MA: Birkhäuser Boston, 2004, pp. 117-141.

[25] D. Edwards, Introduction to Graphical Modelling, ser. Springer Texts in Statistics. Springer New York, 2012.

[26] J. P. Walser, Integer Optimization by Local Search: A Domain-independent Approach. Berlin, Heidelberg: Springer-Verlag, 1999.

[27] V. Berinde, Iterative Approximation of Fixed Points, ser. Lecture Notes in Mathematics. Springer Berlin Heidelberg, 2007.

[28] "The CAIDA anonymized internet traces 2015 dataset," http://www.caida.org/data/passive/passive 2015_dataset.xml, 2015.

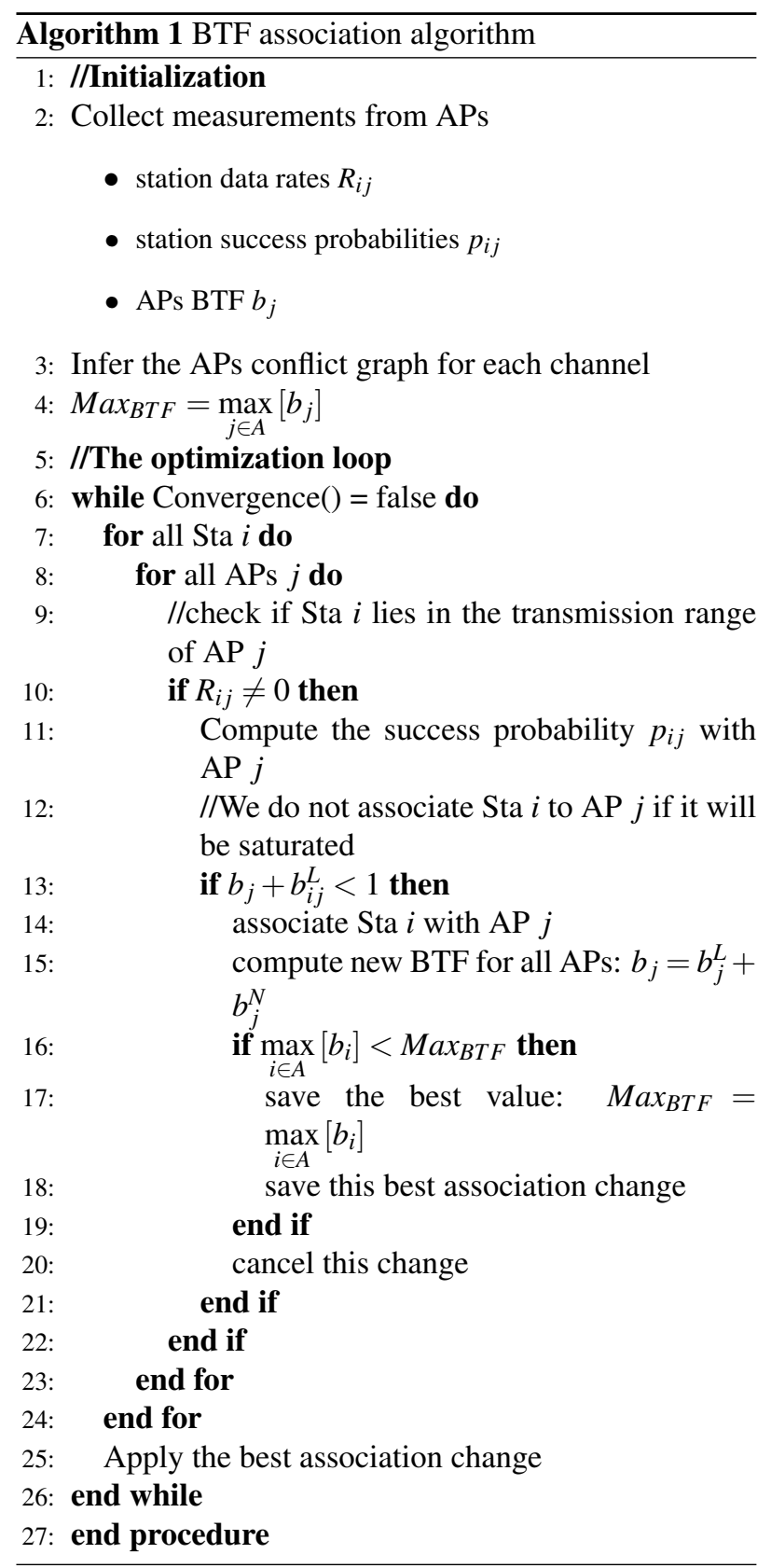

